\title{
Niveles de orégano (Origanum vulgare) en la dieta y su influencia en el rendimiento productivo del pollo de engorde
}

\section{Levels of oregano (Origanum vulgare) in the diet and its influence on the productive performance of the broiler}

\author{
Hilario Pujada Abad ${ }^{1,3}$, Jaime Vega-Vilca ${ }^{1}$, Carlomagno Velásquez Vergara ${ }^{1}$, \\ Betty Palacios-Rodríguez ${ }^{2}$
}

\section{Resumen}

\begin{abstract}
El objetivo del estudio fue determinar el nivel óptimo de orégano (Origanun vulgare) en la dieta alimenticia sobre el rendimiento productivo del pollo de engorde. Se utilizaron 120 pollos machos de un día de edad de la línea Cobb-500, distribuidos al azar en cuatro tratamientos: $0,0.5,1.0$ y $1.5 \%$ de orégano seco y molido en las dietas. Se determinó el peso vivo final, consumo de alimento y conversión alimenticia. Para el análisis de los datos se utilizó el análisis de la varianza de un diseño completamente al azar y para la comparación de medias, la prueba de Tukey. La regresión polinomial y la derivada de una función cuadrática fueron utilizadas para hallar el nivel óptimo de orégano en las variables. Los niveles de orégano empleados influyeron en el peso vivo final, consumo de alimento y conversión alimenticia $(\mathrm{p}<0.05)$, observándose un efecto cuadrático. En promedio, el nivel óptimo de orégano en la dieta fue de $0.71 \%$.
\end{abstract}

Palabras clave: orégano; pollo de engorde; rendimiento; nivel óptimo

\section{Abstract}

The aim of this study was to determine the optimum level of oregano (Origanun vulgare) in the diet on the productive performance of broilers. A total of 1201 -day-old male chickens from the Cobb-500 line were randomly distributed in four treatments: $0,0.5$, 1.0 and $1.5 \%$ of dried and ground oregano in the diets. The final bodyweight, feed

\footnotetext{
${ }^{1}$ Escuela Profesional de Ingeniería Zootécnica, Universidad Nacional José Faustino Sánchez Carrión, Huacho, Perú

${ }^{2}$ Escuela Profesional de Bromatología y Nutrición, Universidad Nacional José Faustino Sánchez Carrión, Huacho, Perú

${ }^{3}$ E-mail: befeen@hotmail.com
}

Recibido: 28 de septiembre de 2018

Aceptado para publicación: 18 de julio de 2019 
consumption and feed conversion were determined. Data was analysed by an analysis of variance through a completely randomized design and the Tukey test for the comparison of means. The polynomial regression and the derivative of a quadratic function were used to find the optimum level of oregano in the variables. The levels of oregano influenced bodyweight, feed consumption and feed conversion $(\mathrm{p}<0.05)$, denoting a quadratic effect. On average, the optimum level of oregano in the diet was $0.71 \%$.

Key words: oregano; broiler; performance; optimum level

\section{INTRODUCCIÓN}

La producción de pollos en el Perú registró un crecimiento de $25 \%$ durante el periodo 2012-2016 debido a la gran demanda de este tipo de carne por parte del público consumidor, que se refleja en el incremento del consumo per cápita nacional de carne de pollo de $48.92 \mathrm{~kg} / \mathrm{hab} / \mathrm{año}$, superando al consumo de carne de bovino de $6.26 \mathrm{~kg} / \mathrm{hab} / \mathrm{año}$ (MINAGRI, 2017).

Los antibióticos usados como promotores de crecimiento en la alimentación de pollos están prohibidos en Europa y EEUU desde 2006 por los efectos adversos de sus residuos en carne sobre la salud del consumidor (DO, 2003). Ante esta situación, se requiere buscar productos alternativos que lo sustituyan. El uso de plantas con propiedades medicinales, como el orégano, romero y laurel, entre otras, puede ser una alternativa de solución para reemplazar a estos promotores de crecimiento.

El orégano (Origanum vulgare) posee aceites esenciales como el carvacrol y timol, que tienen una buena capacidad antioxidante y antimicrobiana contra patógenos como Salmonella typhimurium, Escherichia coli, Staphylococcus aureus y Staphylococcus epidermidis, contribuyendo de esta manera a una mejora en la salud intestinal del ave (Betancourt, 2012; Martínezy Vílchez, 2016). Sin embargo, diversos estudios sobre el uso del orégano como promotores de crecimiento en la alimentación de pollos presentan resultados contradictorios. Por ejemplo, Ayala et al. (2006) y Shiva et al. (2012) no encontraron diferencias en ganancia de peso, consumo de alimento y conversión alimenticia en comparación a los promotores de crecimiento, como los antibióticos; mientras que Valdivia (2016) no encontró diferencias significativas en ganancia de peso, pero sí en consumo de alimento y conversión alimenticia. Al parecer, es necesario profundizar las investigaciones con relación a la dosis de orégano en las dietas alimenticias.

El objetivo de este estudio fue determinar el nivel óptimo de orégano (Origanun vulgare) en la dieta alimenticia sobre el rendimiento productivo del pollo de engorde.

\section{Materiales y Métodos}

El presente trabajo se realizó en el Centro Experimental Avícola de la Escuela Profesional de Ingeniería Zootécnica ubicada dentro del Campus de la Universidad Nacional José Faustino Sánchez Carrión, durante los meses de octubre y noviembre de 2017.

Se utilizaron 120 pollos machos de un día de edad de la línea Cobb 500. Se formaron cuatro grupos al azar de 30 pollos con seis replicaciones cada uno y cinco pollos por réplica: $\mathrm{T}_{0}: 0 \% ; \mathrm{T}_{1}: 0.5 \% ; \mathrm{T}_{2}: 1.0 \% \mathrm{y}_{3}: 1.5 \%$ de orégano seco y molido en la dieta. El alimento fue formulado de acuerdo con las recomendaciones de Cobb-Vantress (s.f.), suministrándose como harina ad libitum, según las fases de producción: pre-inicio (1 a 10 días), crecimiento (23 a 34 días) y acabado 
Cuadro 1. Efecto de los niveles de orégano en la dieta sobre el rendimiento productivo del pollo de engorde (promedio \pm error estándar)

\begin{tabular}{cccc}
\hline $\begin{array}{c}\text { Nivel de } \\
\text { orégano } \\
(\%)\end{array}$ & $\begin{array}{c}\text { Peso final } \\
(\mathrm{g})\end{array}$ & $\begin{array}{c}\text { Consumo alimento } \\
(\mathrm{g})\end{array}$ & $\begin{array}{c}\text { Conversión } \\
\text { alimenticia } \\
(\mathrm{g} / \mathrm{g})\end{array}$ \\
\hline 0.0 & $2732.17 \pm 24.40^{\mathrm{ab}}$ & $4886.70 \pm 33.60^{\mathrm{ab}}$ & $1.79 \pm 0.02^{\mathrm{a}}$ \\
0.5 & $2819.33 \pm 24.40^{\mathrm{a}}$ & $4830.20 \pm 33.60^{\mathrm{bc}}$ & $1.71 \pm 0.02^{\mathrm{b}}$ \\
1.0 & $2815.17 \pm 24.40^{\mathrm{ab}}$ & $4704.70 \pm 33.60^{\mathrm{c}}$ & $1.67 \pm 0.02^{\mathrm{b}}$ \\
1.5 & $2720.67 \pm 24.40^{\mathrm{b}}$ & $4996.70 \pm 33.60^{\mathrm{a}}$ & $1.84 \pm 0.02^{\mathrm{a}}$ \\
\hline
\end{tabular}

$a, b, c$ Superíndices diferentes dentro de columnas indican diferencia estadística $(p<0.05)$

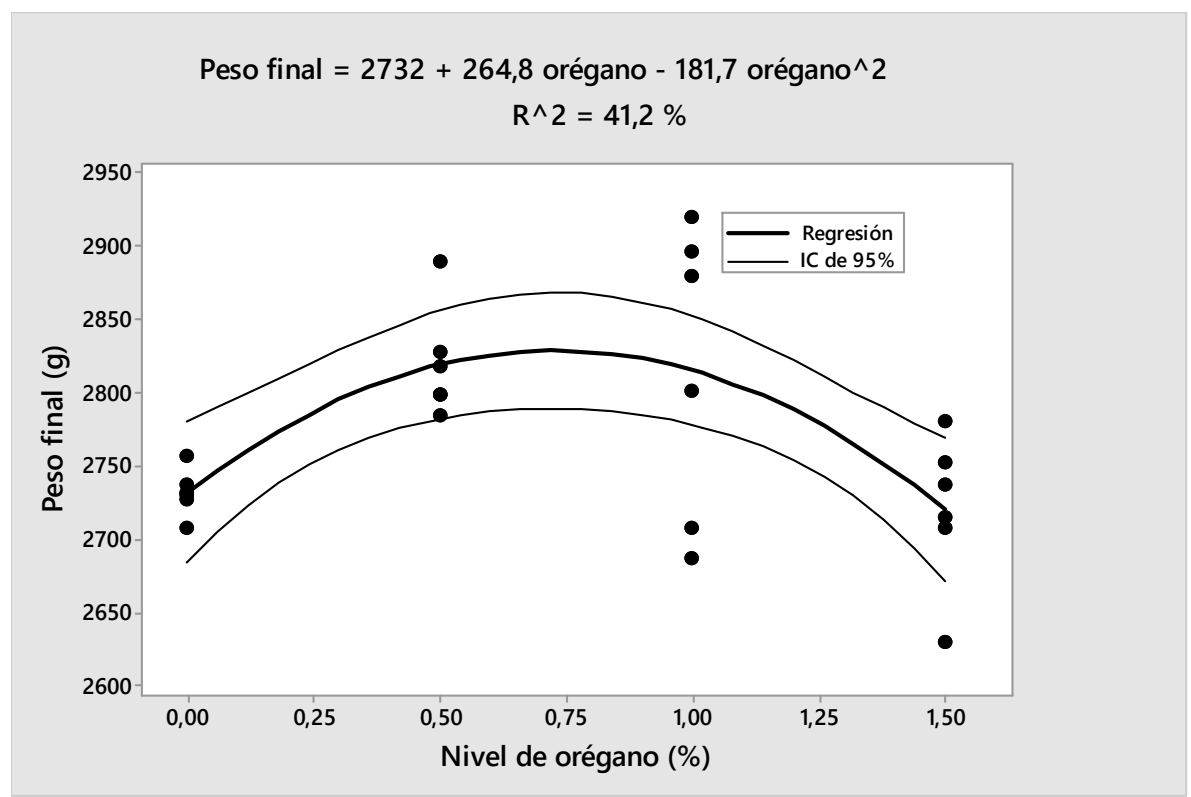

Figura 1. Ajuste al modelo cuadrático del peso final de pollos de carne alimentados con dietas suplementadas con orégano (Origanun vulgare)

(35 a 42 días). Las aves fueron vacunadas en la planta de incubación contra las enfermedades de Marek y Gumboro y en el galpón experimental contra Newcastle, Bronquitis y Gumboro a los 10 y 21 días de edad.

Se evaluó el peso vivo final, el consumo de alimento y la conversión alimenticia, mediante un análisis de varianza con un diseño completamente al azar, previa comprobación de la normalidad y homocedasticidad. Para la comparación entre medias se utilizó la prueba de Tukey (Kuehl, 2001). La regresión polinomial (Little y Hills, 1985) y la derivada de una función cuadrática fueron utilizadas para hallar el nivel óptimo de orégano en cada variable bajo evaluación. 
Cuadro 2. Nivel óptimo de orégano y la predicción del peso final, consumo de alimento y conversión alimenticia de pollos de carne alimentados con dietas suplementadas con orégano (Origanun vulgare), según el modelo cuadrático

\begin{tabular}{lccc}
\hline Variable & $\begin{array}{c}\text { Nivel de orégano } \\
(\%)\end{array}$ & Promedio & IC $(95 \%)^{1}$ \\
\hline Peso final $(\mathrm{g})$ & 0.73 & $2,828.7$ & $2,789-2,868$ \\
Consumo de alimento $(\mathrm{g})$ & 0.69 & $4,744.4$ & $4,677-4,811$ \\
Conversión alimenticia $(\mathrm{g} / \mathrm{g})$ & 0.71 & 1.68 & $1.64-1.71$ \\
\hline
\end{tabular}

${ }^{1}$ Intervalo de confianza al 95\%

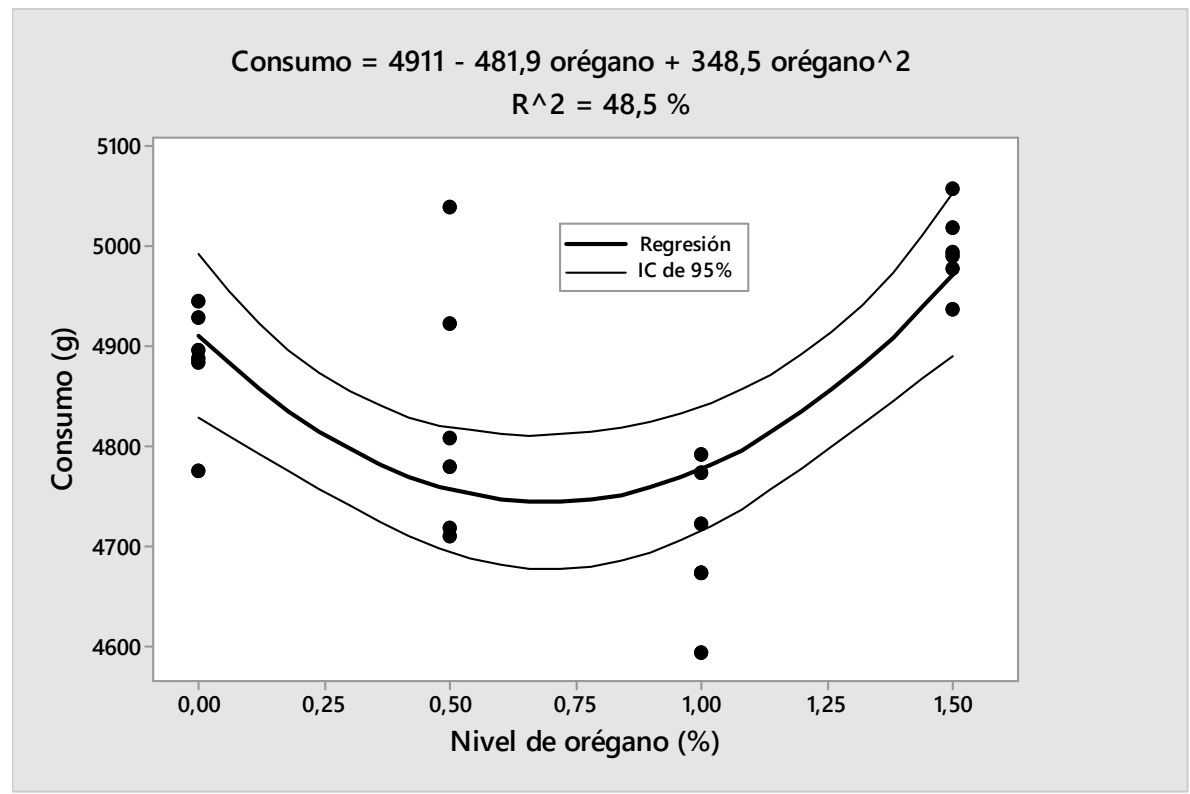

Figura 2. Ajuste al modelo cuadrático del consumo de alimento de pollos de carne alimentados con dietas suplementadas con orégano (Origanun vulgare)

\section{Resultados}

Los niveles de orégano empleados en la dieta influyeron en el peso vivo final, consumo de alimento y conversión alimenticia $(\mathrm{p}<0.05)$, observándose una tendencia cuadrática para estas variables. El peso vivo final aumentó en forma progresiva para finalmente disminuir con el mayor nivel de orégano en la dieta. El comportamiento del con- sumo de alimento y la conversión alimenticia fueron opuestos, pues fueron disminuyendo con el aumento de los niveles de orégano para luego comenzar a incrementar (Cuadro 1).

El ajuste al modelo cuadrático (Figuras 1-3) permitió determinar el nivel óptimo de orégano en la dieta alimenticia para el peso vivo final $(0.73 \%)$, consumo de alimento $(0.69 \%)$ y conversión alimenticia $(0.71 \%)$ (Cuadro 2). Los porcentajes resultantes fue- 
Conversión alimenticia $=1,797-0,3382$ orégano $+0,2395$ orégano^ 2

$R^{\wedge} 2=64,5 \%$

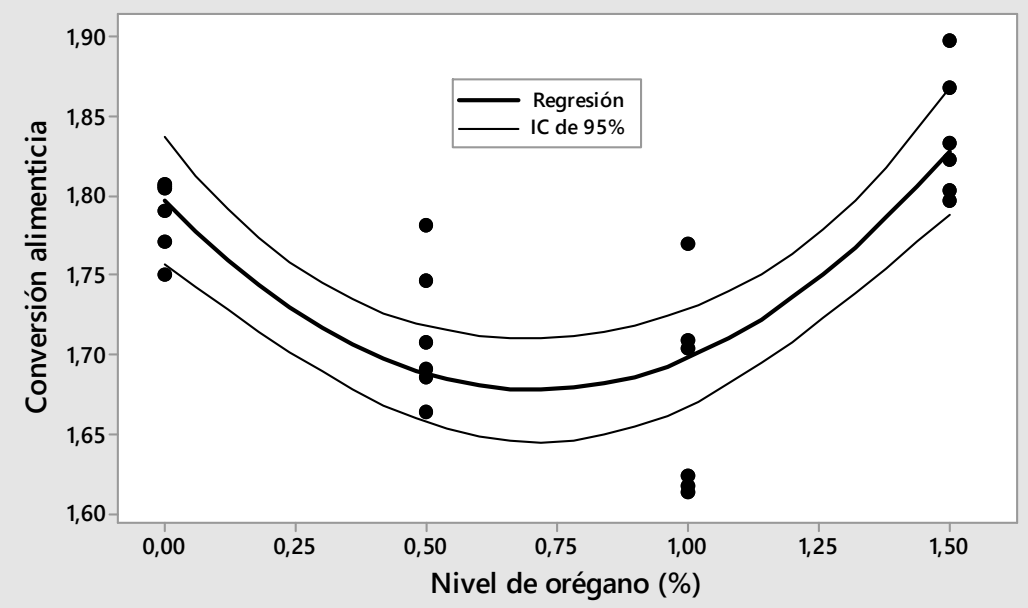

Figura 3. Ajuste al modelo cuadrático de la conversión alimenticia de pollos de carne alimentados con dietas suplementadas con orégano (Origanun vulgare)

ron bastante similares para las tres variables, de allí que se podría considerar que el nivel óptimo de uso estaría en $0.71 \%$.

\section{Discusión}

Los resultados demuestran que la inclusión de niveles crecientes de orégano $(0,0.5$, -1.0 y $1.5 \%)$ en la dieta alimenticia generaron diferencias significativas en el peso vivo final, consumo de alimento y conversión alimenticia $(\mathrm{p}<0.05)$ en pollos de carne a los 42 días de edad. Los resultados difieren de los trabajos de Ayala et al. (2006) y Valdivia (2016), quienes no encontraron diferencias significativas en peso vivo, aunque si obtuvieron en consumo de alimento y conversión alimenticia, mientras que otros autores no encontraron diferencias significativas en las tres variables evaluadas (Botgoglou et al., 2002; Shiva et al., 2012; Chiriboga, 2014). Estas diferencias podrían deberse a la especie, dosis y forma de suministro del orégano, así como a los métodos empleados para determinar su actividad (Garcés et al., 2009).
Se observó un comportamiento cuadrático con los niveles crecientes de orégano administrados en la dieta. El nivel de $1.5 \%$ de orégano $\left(\mathrm{T}_{3}\right)$ generó una menor respuesta en el peso vivo e incrementó el consumo de alimento, y como consecuencia se elevó la conversión alimenticia. Estos resultados coinciden con lo obtenido por Betancourt (2012), quienes mencionan un efecto deletéreo en el comportamiento productivo de pollos de engorde con dosis altas de orégano. Según Betancourt (2012), el efecto negativo estaría asociado a los niveles elevados de timol y carvacrol presentes en el orégano y que serían tóxicos, afectando la salud intestinal, manifestándose con un menor peso corporal en los pollos de engorde.

El nivel óptimo promedio de orégano en la dieta alimenticia obtenido mediante el modelo cuadrático fue de $0.71 \%$, encontrándose dentro del rango porcentual utilizado por Ayala et al (2006), quienes obtuvieron un aumento en el consumo de alimento con la dosis de $1 \%$. 


\section{ConClusiones}

- Niveles de $0,0.5,1.0$ y $1.5 \%$ de orégano (Origanun vulgare) en las dietas alimenticias de pollos de engorde mostraron un efecto cuadrático en las variables peso vivo final, consumo de alimento y conversión alimenticia.

- El nivel óptimo de uso del orégano en la dieta alimenticia fue de $0.71 \%$, obteniéndose un mayor peso vivo final, mejor conversión alimenticia y menor consumo de alimento.

\section{Literatura Citada}

1. Ayala L, Martínez M, Acosta A, Dieppa O, Hernández L. 2006. Una nota acerca del efecto del orégano como aditivo en el comportamiento productivo de pollos de Ceba. Cuban J Agr Sci 40: 455-458.

2. Betancourt LL. 2012. Evaluación de aceites esenciales de orégano en la dieta de pollos de engorde. Tesis Doctoral. Bogotá: Univ. Nacional de Colombia. 157p.

3. Botsoglou NA, Florou-Paneri P, Christaki E, Fletouris DJ, Spais AB. 2002. Effect of dietary oregano essential oil on performance of chickens and on iron-induced lipid oxidation of breast, thigh and abdominal fat tissues. Brit Poultry Sci 43: 223-230. doi: 10.1080/00071660120121436

4. Chiriboga CL. 2014. Utilización de vinagre (ácido acético) e infusión de orégano (Plectranthus amboinicus) como prebiótico en el levante de pollos criollos Gallus domesticus tipo mejorados. Tesis de Médico Veterinario y Zootecnista. Ecuador: Univ. Técnica de Machala. $64 \mathrm{p}$.

5. Cob-Vantres. s.f. Broiler Cobb500 Broiler performance \& nutrition supplement. [Internet]. Available in: https://www.cobb-vantress.com/assets/ Cobb-Files/product-guides/bdc20a5443/ 70 d e c $630-0$ a b f - 11 e $9-9$ c 88 c51e407c53ab.pdf

6. [DO] Diario Oficial de la Unión Europea. 2003. Reglamento (CE) N. ${ }^{\circ}$ 1831/2003 sobre los aditivos en la alimentación animal. [Internet]. Disponible en: https://www.boe.es/doue/2003/268/ L00029-00043.pdf

7. Garcés C, Soler M, Barragán J. 2009. ¿Ejercen los extractos vegetales un efecto positivo sobre broilers enfermos? Albéitar 130: 18-20.

8. Kuehl RO. 2001. Diseño de experimentos: principios estadísticos de diseño y análisis de investigación. $2^{\text {a }}$ ed. México: Thomson Learning. $680 \mathrm{p}$.

9. Little TM, Hills FJ. 1985. Métodos estadísticos para la investigación en la agricultura. México: Trillas. $270 \mathrm{p}$.

10. Martínez D, Vílchez C. 2016. Oregano essential oil improves nutrient absorption capacity in healthy broilers. Poult Sci 95(Suppl. 1): 217.

11. [MINAGRI] Ministerio de Agricultura y Riego. 2017. Anuario estadístico de la producción pecuaria y avícola 2017. [Internet]. Disponible en: http:// siea.minagri.gob.pe/siea/?q=noticias/ anuario-estadistico-producccion-pecuaria-y-avicola-2017

12. Shiva C, Bernal S, Sauvain M, Caldas J, Kalinowski J, Falcón N, Rojas R. 2012. Evaluación del aceite esencial de orégano (Origanum vulgare) y extracto deshidratado de jengibre (Zingiber officinale) como potenciales promotores de crecimiento en pollos de engorde. Rev Inv Vet Perú 23: 160-170. doi: 10.15381/rivep.v23i2.896

13. Valdivia E. 2016. Evaluación de tres niveles de orégano (Origanum vulgare $\mathrm{L}$ ) como aditivo en la alimentación en dos etapas de producción en pollos parrilleros Tesis de Ingeniero Agrónomo. Bolivia: Universidad Mayor de San Andrés. 128 p. 